\section{Experiências com estágios externos na Residência de Medicina de Família e Comunidade da Faculdade de Medicina da Universidade de São \\ Paulo}

Experiences with external clerkships at the Family and Community Medicine Residency of the Medical School of the University of São Paulo

\author{
Experiencias con prácticas externas en la residencia de \\ Medicina de Familia y Comunidad de la Facultad de Medicina \\ de la Universidad de São Paulo
}

\section{Resumo}

Introdução: A formação do médico de família e comunidade tem nos serviços de Atenção Primária à Saúde o principal cenário de práticas. Além destes, outros estágios têm sido empregados para o aprimoramento de competências específicas. Objetivo: Descrever o perfil de estágios externos realizados pelos médicos residentes no programa de residência em Medicina de Família e Comunidade da Faculdade de Medicina da Universidade de São Paulo. Resultados: Os estágios externos acontecem durante 2 meses no segundo ano de residência e são divididos igualmente em estágio eletivo (em que é necessário vínculo com a instituição) e optativo (podem ser selecionados livremente). A maioria destes estágios ocorre no Brasil e através de vínculos informais, sem haver uma parceria ou arranjo oficial entre instituições e/ou serviços. Os principais estágios buscados são em medicina rural e no National Health Service (Reino Unido). Conclusão: Estágios externos enriquecem a formação do residente e possibilitam o aprimoramento do programa de residência. Grande parte dos estágios é solicitada por contato informal, o que aponta para o desafio de firmar vínculos entre pessoas, serviços e instituições, visando fortalecer cada vez mais a formação em Medicina de Família e Comunidade.

Palavras-chave: Educação Médica; Estágio Clínico; Internato e Residência; Medicina de Família e Comunidade; Atenção Primária à Saúde

\author{
Gustavo Kang Hong Liua \\ Marcus Vinícius Camargo Garcia de Pontes ${ }^{a}$ \\ Kamila Vieira Silvab \\ Tatiana Milla Mandia ${ }^{c}$ \\ Bianca Luiza de Sá e Silva ${ }^{a}$ \\ José Benedito Ramos Valladão Júniora
}

\begin{abstract}
a Programa de Residência em Medicina de Família e Comunidade da Faculdade de Medicina da Universidade de São Paulo (FMUSP). São Paulo, SP, Brasil. gustavoliu@gmail.com (Autor correspondente); mvcgpontes@gmail.com; biluiza@ hotmail.com; josevalladao@gmail.com

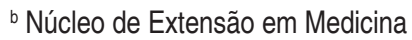
Tropical da Universidade de São Paulo (NUMETROP/USP); Universidade do Estado do Pará (UEPA). Santarém, PA, Brasil. kvs.vieira@gmail.com

c Faculdade de Medicina da Universidade de São Paulo (FMUSP). São Paulo, SP, Brasil. tatimandia@ hotmail.com
\end{abstract}

Como citar: Liu GKH, Pontes MVCG, Silva KV, Mandia TM, Silva BLS, Valladão Júnior JBR. Experiências com estágios externos na Residência de Medicina de Família e Comunidade da Faculdade de Medicina da Universidade de São Paulo. Rev Bras Med Fam Comunidade. 2018;13(40):1-7. http://dx.doi.org/10.5712/rbmfc13(40)1608
Fonte de financiamento: declaram não haver.

Parecer CEP: não se aplica. Conflito de interesses: declaram não haver. Procedência e revisão por pares: revisado por pares. Recebido em: 30/08/2017. Aprovado em: 03/02/2018. 


\begin{abstract}
Introduction: The main field of training of the Family Doctor are Primary Health Care services as primary practice scenario. In addition to these, other stages are used to enhance the learning of specific competencies. Objective: To describe the profile of external internships performed by residents in the Residency Program in Family Medicine of the Medical School of the University of São Paulo. Results: External internships are held for 2 months in the second year of residency and are equally divided into elective internships (in which a bond with the institution is required) and optional (freely selectable). Most of these internships occur in Brazil and through informal ties, without a partnership or official arrangement between institutions and/or services. The main stages sought are in rural medicine and the National Health Service (UK). Conclusion: The external internships enriches the resident's training and enable the improvement of the residency program. Most of the internships are requested through informal contact, which points to the challenge of establishing links between people, services and institutions to strengthen training in Family and Comunnity Medicine.
\end{abstract}

Keywords: Medical Education; Clinical Clerkship; Internship and Residency; Family Practice; Primary Health Care

\title{
Resumen
}

Introducción: La formación del médico de familia y comunidad tiene en los servicios de Atención Primaria a la Salud el principal escenario de prácticas. Además de éstas, otras prácticas se emplean para perfeccionar competencias específicas. Objetivo: Describir el perfil de prácticas externas realizadas por los médicos residentes en el programa de residencia en Medicina de Familia y Comunidad de la Facultad de Medicina de la Universidade de São Paulo. Resultados: Las practicas externas ocurren durante 2 meses en el segundo año de residencia y se dividen igualmente en las prácticas electivas (en las que es necesario un vínculo con la institución) y optativas (se pueden seleccionar libremente). La mayoría de estas prácticas ocurre en Brasil y a través de vínculos informales, sin que haya una sociedad o arreglo oficial entre instituciones y/o servicios. Las principales etapas buscadas son en medicina rural y en el National Health Service (Reino Unido). Conclusión: Las prácticas externas enriquecen la formación del residente y posibilitan el perfeccionamiento del programa de residencia. Gran parte de las prácticas es seleccionada por contacto informal, lo que apunta el desafío de firmar vínculos entre personas, servicios e instituciones visando fortalecer cada vez más la formación en Medicina de Familia y Comunidad.

Palabras clave: Educación Médica; Prácticas Clínicas; Internado y Residencia; Medicina Familiar y Comunitaria; Atención Primaria de Salud

\section{Introdução}

A formação do médico de família e comunidade tem como principal campo os serviços de Atenção Primária à Saúde (APS), aprendendo e praticando as competências necessárias para sua atuação com excelência, em estágios supervisionados. Além desses estágios principais na APS, estágios em outros serviços são utilizados para o aprimoramento de competências específicas e estímulo à reflexão sobre a rede de cuidados em saúde. Tais estágios têm cenários mais diversos, ocorrendo em contextos de atenção primária, secundária ou terciária à saúde ou de interface com a rede de cuidados em saúde, como, por exemplo, a gestão em saúde ou metodologia científica. ${ }^{1,2}$

Em alguns programas de residência no Brasil e no mundo, os estágios podem estar previamente estabelecidos no planejamento da formação do médico de família e comunidade ou podem haver espaços livres onde é possível a seleção de estágios para aprimoramento de áreas de interesse do residente e complementação do aprendizado de competências específicas. ${ }^{2-6}$

\section{Desenvolvimento}

Esse artigo pretende apresentar os modelos de estágio externos realizados no Programa de Residência Médica em Medicina de Família e Comunidade (PRM-MFC) da Faculdade de Medicina da Universidade de São Paulo (FMUSP), suas características e o perfil de procura pelos médicos residentes. A partir destas observações, são realizadas reflexões a respeito da importância dos estágios e da articulação entre serviços para o fortalecimento da especialidade e da qualificação da Atenção Primária à Saúde no país. 


\section{Modelo de estágios externos}

No PRM-MFC da FMUSP, os médicos residentes realizam estágios de intercâmbio nacionais ou internacionais eletivos e optativos durante o segundo ano de residência. São chamados de eletivos os estágios que podem ser selecionados pelo residente e estão vinculados ao serviço ao qual está alocada a Comissão de Residência Médica (COREME) do programa de residência e de optativos os estágios que podem ser selecionados pelo residente sem a limitação de estarem vinculados ao serviço que aloca a COREME.

Os estágios podem tanto ocorrer em cenários de prática de atenção primária à saúde quanto em áreas de interesse específicas dos residentes (infectologia, fisiatria, cuidados paliativos, ensino, gestão, etc.).

São destinados 30 dias corridos para realização de estágios eletivos e o mesmo tempo para a realização de estágios optativos. Respeitando este espaço de tempo delimitado, os residentes podem dividir este ínterim em períodos menores para realização de diferentes estágios. Os residentes selecionam os estágios a partir de seus próprios interesses didáticos, sendo solicitada aprovação por parte da coordenação do programa de residência.

Como é respeitada certa liberdade na procura pelos estágios externos, os médicos residentes realizam diferentes estratégias para conseguirem realizá-los dentro de suas preferências pessoais e plausibilidade formativa para aprovação do PRM. Temos visto que, de forma geral, o contato inicial para a realização desses estágios pode ocorrer de maneira formal ou informal. Os estágios estabelecidos através de vínculos formais são firmados por meio de uma solicitação estruturada e oficial de estágio entre o programa de residência médica e o serviço de destino, podendo ou não existir parceria interinstitucional. Os estágios realizados através de vínculo informal ocorrem quando, por outro lado, não há um fluxo organizado e definido para a sua solicitação. Estes estágios, habitualmente, são construídos pelo contato que partiu do próprio residente ou por parte de um conhecido que intermediou o pedido.

Além disso, os estágios escolhidos pelos residentes podem apresentar o perfil de atividades observacionais e/ou práticas.

Nos estágios observacionais, o residente busca a aquisição de conhecimentos sobre modelos de sistema de saúde, de organização da APS, de gestão da clínica na MFC no Brasil e no mundo, bem como acompanhar e absorver conhecimentos para incorporar à sua prática pessoal a partir da observação de consultas de profissionais mais experientes.

Nos estágios práticos, o residente busca o desenvolvimento de competências técnicas e habilidades clínicas em serviços reconhecidos a partir de necessidades específicas que ele entenda que deve pessoalmente aprimorar.

A seguir, organizamos as experiências em estágios externos que o PRM-MFC da FMUSP tem realizado desde 2013. O Quadro 1 enumera os estágios externos nacionais e o Quadro 2, os estágios internacionais.

\section{Discussão}

O modelo de estágios externos relatado permite que o aprendizado dos residentes esteja de acordo com suas necessidades e interesses individuais, o que é preconizado pela WONCA (World Organization of Family Doctors) ${ }^{5}$ e em programas de residência de MFC do mundo todo, como Portugal e Espanha..$^{6-8}$ No 
Quadro 1. Estágios Externos Nacionais realizados no Programa de Residência em Medicina de Família e Comunidade da Faculdade de Medicina da Universidade de São Paulo (FMUSP).

\begin{tabular}{|c|c|c|c|}
\hline Estágio & Cenário & $\begin{array}{l}\text { Forma de contato para } \\
\text { solicitação do estágio }\end{array}$ & $\begin{array}{c}\text { Atividades } \\
\text { desempenhadas }\end{array}$ \\
\hline $\begin{array}{l}\text { Serviços de APS da SMS da Cidade do Rio de } \\
\text { Janeiro - RJ }\end{array}$ & MFC na APS & Informal & Observacionais \\
\hline $\begin{array}{l}\text { Programa de Residência de MFC do Hospital Odilon, } \\
\text { Belo Horizonte - MG }\end{array}$ & MFC na APS & Informal & Observacionais \\
\hline $\begin{array}{l}\text { Programa de Residência de MFC do Grupo } \\
\text { Hospitalar Conceição - RS }\end{array}$ & MFC na APS & Informal & Observacionais \\
\hline $\begin{array}{l}\text { Serviços de APS da SMS da Cidade de } \\
\text { Florianópolis - SC }\end{array}$ & MFC na APS & Informal & Observacionais \\
\hline Hospital Municipal de Santarém - PA & Infectologia & Formal & Práticas \\
\hline $\begin{array}{l}\text { Serviço de APS da SMS de Santarém em Alter do } \\
\text { Chão - PA }\end{array}$ & MFC na APS & Formal & Práticas \\
\hline $\begin{array}{l}\text { Serviços de Gestão da SMS da Cidade de } \\
\text { Florianópolis - SC }\end{array}$ & Gestão & Informal & Observacionais \\
\hline Serviço de APS da SMS de Cajazeiras - PB & MFC na APS & Informal & Observacionais \\
\hline Serviço de APS da SMS de Petrolina - PE & MFC na APS & Informal & Observacionais \\
\hline Residência de MFC de São Bernardo do Campo - SP & MFC na APS & Informal & Observacionais \\
\hline Hospice Jaçanã - Cuidados Paliativos & Cuidados Paliativos & Informal & Práticas \\
\hline Consultório na Rua em Rio de Janeiro - RJ & MFC na APS & Informal & Observacionais \\
\hline Consultório na Rua em Campinas - SP & MFC na APS & Informal & Observacionais \\
\hline $\begin{array}{l}\text { Institutos de Medicina Física e Reabilitação do } \\
\text { Hospital das Clínicas da FMUSP }\end{array}$ & Fisiatria & Formal & Observacionais \\
\hline $\begin{array}{l}\text { Casa da AIDS da Divisão de Moléstias Infecciosas e } \\
\text { Parasitárias do Hospital das Clínicas da FMUSP }\end{array}$ & Infectologia & Formal & Práticas \\
\hline $\begin{array}{l}\text { Serviço de Geriatria do Hospital das Clínicas da } \\
\text { FMUSP }\end{array}$ & Geriatria & Formal & Observacionais \\
\hline Projeto de Extensão Universitária Bandeira Científica & Ensino e MFC na APS & Formal & Práticas \\
\hline $\begin{array}{l}\text { Centro de Referência de Álcool, Tabaco e outras } \\
\text { Drogas de São Paulo - SP }\end{array}$ & Saúde Mental & Informal & Observacionais \\
\hline $\begin{array}{l}\text { Grupo de Dor - Serviço de Neurologia do Hospital } \\
\text { das Clínicas da FMUSP }\end{array}$ & Neurologia & Formal & Observacionais \\
\hline $\begin{array}{l}\text { Coordenação de Epidemiologia e Informação da } \\
\text { SMS de São Paulo - SP }\end{array}$ & Gestão & Formal & Observacionais e Práticas \\
\hline $\begin{array}{l}\text { Coordenação de Atenção Básica da SMS de São } \\
\text { Paulo - SP }\end{array}$ & Gestão & Informal & Observacionais \\
\hline $\begin{array}{l}\text { Escola Municipal de Saúde e Supervisão Técnica de } \\
\text { Saúde da SMS de São Paulo }\end{array}$ & Gestão & Informal & Observacionais \\
\hline
\end{tabular}

SMS: Secretaria Municipal de Saúde.

Brasil, temos como exemplos o programa de residência em Medicina de Família e Comunidade do Serviço de Saúde Comunitária do Grupo Hospitalar Conceição (Porto Alegre/RS), em que os residentes têm 2 meses para realizar estágio optativo, ${ }^{3}$ e o programa de residência em Medicina de Família e Comunidade da Secretaria Municipal de Saúde do Rio de Janeiro, em que o residente tem possibilidade de realizar estágio optativo durante um mês. ${ }^{4}$

A existência deste modelo no programa de residência de MFC da FMUSP é possível porque os residentes não são os únicos responsáveis pela assistência médica à população do serviço de APS onde 
Quadro 2. Estágios Externos Internacionais realizados no Programa de Residência de Medicina de Família e Comunidade da Faculdade de Medicina da Universidade de São Paulo (FMUSP).

\begin{tabular}{|c|c|c|c|}
\hline Estágio & Cenário & $\begin{array}{l}\text { Forma de contato para } \\
\text { solicitação do estágio }\end{array}$ & $\begin{array}{c}\text { Atividades } \\
\text { desempenhadas }\end{array}$ \\
\hline $\begin{array}{l}\text { Department of Primary Care and Public Health Sciences do } \\
\text { King's College London (Inglaterra) }\end{array}$ & MFC na APS e Ensino & Formal & Observacionais \\
\hline King's College Hospital (Inglaterra) & Cuidados Paliativos & Formal & Observacionais \\
\hline $\begin{array}{l}\text { Centros de Atenção Primária em Barcelona, Granada e Madri } \\
\text { (Espanha) }\end{array}$ & MFC na APS & Informal & Observacionais \\
\hline $\begin{array}{l}\text { Centros de Atenção Primária em Barreiro, Lisboa e Porto } \\
\text { (Portugal) }\end{array}$ & MFC na APS & Formal & Observacionais \\
\hline $\begin{array}{l}\text { Centros de Atenção Primária em Bristol, Londres e Surrey } \\
\text { (Inglaterra) }\end{array}$ & MFC na APS & Informal & Observacionais \\
\hline Centros de Atenção Primária em Charleroi e Liége (Bélgica) & MFC na APS & Informal & Observacionais \\
\hline Centros de Atenção Primária em Amsterdam e Zeist (Holanda) & MFC na APS & Formal & Observacionais \\
\hline Centro de Atenção Primária em Santa Clara (Cuba) & MFC na APS & Informal & Observacionais \\
\hline Centro de Atenção Primária em Adelaide (Austrália) & MFC na APS & Informal & Observacionais \\
\hline Centro de Atenção Primária em Nova Deli (Índia) & MFC na APS & Formal & Observacionais \\
\hline Centro de Atenção Primária em Banyuls sur Mer (França) & MFC na APS & Formal & Observacionais \\
\hline Centro de Atenção Primária em Avezzano (Itália) & MFC na APS & Formal & Observacionais \\
\hline
\end{tabular}

estão inseridos. Os residentes são inseridos em equipes de Estratégia de Saúde da Família (ESF) em que há um especialista em MFC, o qual é denominado "tutor", cuja participação no processo de aprendizado é primordial.

Este tutor é o responsável por toda a demanda assistencial médica em momentos de ausência do residente de sua equipe, modelo também repetido em programas de residência de países com sistemas de saúde orientados pela APS.$^{6,7}$ Este fato garante que o atendimento à população não seja comprometido durante os períodos de estágios externos e férias regulares do médico residente.

Em nossa experiência, como ilustrado nos Quadros 1 e 2, podemos verificar uma diversidade de estágios externos que vêm sendo realizados desde 2013. Os estágios nacionais são os mais procurados, possivelmente devido a facilidades como idioma, menor custo financeiro e deslocamento. Todavia, também percebemos uma procura considerável por estágios internacionais, majoritariamente concentrada no continente europeu, provavelmente pela grande carga de importância, desenvolvimento e investimento que os sistemas de saúde nesta região do globo têm prestado à especialidade ao longo dos anos.

Os cenários de prática de estágios externos nacionais e internacionais têm ocorrido principalmente em serviços de APS. Quando não realizados em serviços de APS, notamos a procura por serviços nas seguintes áreas de interesse: cuidados paliativos, infectologia, geriatria, fisiatria, neurologia, saúde mental, gestão e ensino.

Temos observado que a maioria dos residentes de nosso PRM têm procurado estágios práticos em medicina rural, por ser uma das competências de atuação do MFC deficiente em nosso PRM por estarmos localizados em uma metrópole, sem cenários de práticas ou experiência com esta área do cuidado em saúde. Devido a esta deficiência formativa, fundou-se um vínculo institucional bem estabelecido com a Unidade de Saúde de Alter do Chão (Santarém/PA). Este estágio externo em medicina rural se destaca por ocorrer em uma unidade cuja médica da ESF é especialista em MFC egressa do nosso programa de 
residência e quem supervisiona os estagiários em atendimento de livre demanda, visitas domiciliares na vila e em comunidades vizinhas (povo ribeirinho, de mata e floresta).

Neste período, há possibilidade de contato com atendimento a pessoas com doenças crônicas; condições de urgência e emergência; doenças infecciosas tropicais como, por exemplo, malária, leishmaniose e hanseníase; vítimas de acidentes com animais peçonhentos, e realização de procedimentos em pequenas cirurgias. Soma-se a toda essa carga prática uma vivência transcultural em medicina rural, fruto do estagiário trabalhar e residir, temporariamente, na vila em meio à floresta amazônica, imerso nesta comunidade, participando de problemáticas vividas diariamente pela população local, permitindo uma identificação marcante da necessidade do médico de família e comunidade adquirir competências culturais, econômicas, políticas e sociais da comunidade em que atua; enriquecendo sua formação com esta experiência e proporcionando um estímulo à possibilidade de interiorização da MFC no Brasil.

Os estágios observacionais também têm se mostrado de grande validade para amadurecimento e definição identitária do médico residente sobre sua prática e trazido grandes retornos aos serviços de APS de nosso PRM. Um exemplo de como isso aconteceu é no processo de abertura do acesso nas unidades em que ocorre o ensino. O programa conta atualmente com cinco unidades básicas de saúde que recebem residentes, sendo que, hoje, todas realizam alguma forma de agendamento de pacientes para o mesmo dia. Pode-se afirmar que a experiência com estágios externos contribuiu para o processo de abertura do acesso nestas unidades nos últimos anos, pois muitos egressos do programa se tornam médicos na assistência e ensino nestas unidades. O fato de conhecer outros contextos e fluxos de trabalho, por exemplo, de serviços da Europa, onde o acesso avançado é praticado, embasou os profissionais envolvidos no sentido de propor e ajudar na implementação destas mudanças.

Dentre as dificuldades e desafios que podemos apontar para maior consolidação e aprimoramento dos estágios externos, nota-se que, para a realização de muitos dos estágios, o vínculo se estabelece de forma informal, partindo do próprio residente ou realizado por alguém vinculado à residência que conhecia um profissional no serviço de destino. Dada a natureza diversa dos interesses individuais dos residentes e da grande quantidade de serviços existentes no mundo, é esperado que sempre exista uma porção de novos contatos sendo realizados para solicitação de estágios de maneira informal, e que apenas os serviços já conhecidos, com o passar do tempo, tenham estabelecido formas de intercâmbio melhor estruturadas.

Dada a relevância dos estágios externos como experiência formativa e para fortalecimento do trabalho na APS, gostaríamos de ressaltar a importância que oportunidades de estabelecer vínculos e contatos têm para a qualificação da especialidade e da rede como um todo. Dentre estas, destacamos duas: no âmbito nacional, eventos da especialidade, e, no internacional, o programa WONCA Family Medicine $360^{\circ}\left(\mathrm{FM} 360^{\circ}\right) .{ }^{9}$ Eventos como o Congresso Brasileiro de Medicina de Família e Comunidade têm sido um espaço rico para trocas de experiências e o estabelecimento de contatos chave para a colaboração, o que inclui a realização de estágios interinstitucionais.

O FM360', por sua vez, é um programa de intercâmbio iniciado em 2013 com o apoio dos movimentos de jovens médicos da WONCA. Tem como objetivo conectar médicos que tenham até cinco anos de prática de MFC com anfitriões em serviços de saúde em outros países para realização de visitas observacionais de até quatro semanas, e tem sido uma ponte importante entre os residentes de nosso programa e diversos serviços de saúde no exterior. 


\section{Conclusão}

O oferecimento de estágios externos é importante para que se complemente o aprendizado de forma a respeitar as necessidades e interesses específicos de cada residente. Através da incorporação do conhecimento e prática de outros serviços de APS e outros níveis de atenção à saúde, a experiência adquirida em estágios externos nacionais e internacionais contribui também para o enriquecimento do programa de residência e qualificação da rede assistencial em que ele está inserido.

Muitos estágios dependem de contatos informais para sua realização, o que aponta para o desafio de fortalecer os vínculos entre pessoas, serviços e instituições para oferecer oportunidades de melhorar cada vez mais a formação em MFC. ${ }^{10}$ A participação em eventos da especialidade (conferências, congressos, encontros, etc.) e o programa WONCA FM360 são formas relevantes para melhorar esta aproximação.

\section{Referências}

1. Augusto DK. Residência em Medicina de Família e Comunidade. In: Gusso G, Lopes JMC. Tratado de Medicina de Família e Comunidade: Princípios, Formação e Prática. Porto Alegre: Artmed; 2012. p. 381-6.

2. Brasil. Secretaria de Educação Superior. Resolução $n^{\circ}$ 1, de 25 de Maio de 2015. Regulamenta os requisitos mínimos dos programas de residência médica em Medicina Geral de Família e Comunidade - R1 e R2 e dá outras providências. Diário Oficial da União. 2015 Mai 26 ; Seção 1. [Acesso 2018 Fev 01]. Disponível em: http://portal.mec.gov.br/index.php?option=com_docman\&view=download\&alias=20741 res01-25052015-cnrm-regulamenta-requisitos-pdf\&category_slug=setembro-2015-pdf\&ltemid=30192

3. Castro RCL, Diercks MLMS, Corrêa FA, Gyrão NB, Pekelman R, Umpierre RN. O programa teórico da residência em Medicina de Família e Comunidade do Serviço de Saúde Comunitária do Grupo Hospitalar Conceição, Porto Alegre, RS. Rev Bras Med Fam Comunidade. 2014;9(33):375-83. DOI: http://dx.doi.org/10.5712/rbmfc9(33)855

4. Justino ALA, Oliver LL, Melo TP. Implantação do Programa de Residência em Medicina de Família e Comunidade da Secretaria Municipal de Saúde do Rio de Janeiro, Brasil. Ciênc Saúde Coletiva [Internet]. 2016 [citado 2018 Fev 01]; 21(5):1471-80. DOI: http://dx.doi. org/10.1590/1413-81232015215.04342016

5. WONCA Working Party on Education. WONCA global standards for postgraduate family medicine education. [Internet] 2013. [citado 2018 Fev 01]. Disponível em: http://www.globalfamilydoctor.com/site/DefaultSite/filesystem/documents/Groups/Education/WONCA\%20 ME\%20stds_edit\%20for\%20web_250714.pdf

6. Costa A, Pedro R, Magalhães S. Medicina Geral e Familiar em Portugal. Rev Bras Med Fam Comunidade. 2014;9(32):295-9. DOI: http:// dx.doi.org/10.5712/rbmfc9(32)935

7. Casado V, Luzón L. Razones, retos y aportaciones del programa de la especialidad de Medicina Familiar y Comunitaria en España. Rev Bras Med Fam Comunidade. 2014;9(31):195-205. DOI: http://dx.doi.org/10.5712/rbmfc9(31)876

8. Coordenação do Internato Médico de Medicina Geral e Familiar de Lisboa e Vale do Tejo. Manual de Formação Versão 2018.a [Internet]. 2018 Jan 09. [citado 2018 Jan 30] Disponível em: http://www.arslvt.min-saude.pt/uploads/writer_file/document/5282/Manual_de_ Formac_a_o_2018-a.pdf

9. Family Medicine $360^{\circ}\left(\mathrm{FM} 360^{\circ}\right)$ webpage. [Internet] [citado $2018 \mathrm{Fev} 01$ ]. Disponível em: https://sites.google.com/site/familymedicine360/ home

10. Evensen A, Wondimagegn D, Zemenfes Ashebir D, Rouleau K, Haq C, Ghavam-Rassoul A, et al. Family Medicine in Ethiopia: Lessons from a Global Collaboration. J Am Board Fam Med. 2017;30(5):670-7. DOI: http://dx.doi.org/10.3122/jabfm.2017.05.170086 